Revue d'histoire de l'Amérique française

ZAS REVUE D.HISTOIRE DE L'AMÉRIQUE FRANÇAISE

\title{
Codicille au testament olographe de Lamothe Cadillac
} $18^{\mathrm{e}}$ juillet 1705

\section{Lamothe Cadillac}

Volume 3, numéro 3, décembre 1949

URI : https://id.erudit.org/iderudit/801581ar

DOI : https://doi.org/10.7202/801581ar

Aller au sommaire du numéro

Éditeur(s)

Institut d'histoire de l'Amérique française

ISSN

0035-2357 (imprimé)

1492-1383 (numérique)

Découvrir la revue

Citer ce document

Cadillac, L. (1949). Codicille au testament olographe de Lamothe Cadillac : $18^{\mathrm{e}}$ juillet 1705. Revue d'histoire de l'Amérique française, 3(3), 447-448.

https://doi.org/10.7202/801581ar d'utilisation que vous pouvez consulter en ligne.

https://apropos.erudit.org/fr/usagers/politique-dutilisation/ 


\title{
DOCUMENTS INÉDITS
}

\author{
CODICILLE AU TESTAMENT OLOGRAPHE DE \\ LAMOTHE CADILLAC \\ $18^{\circ}$ juillet $1705^{*}$
}

Ce jourdhui dix huitieme Juillet 1705 moi antoine de Lamothe Cadillac capitaine et comandant pour le roi du fort pontchartrain du detroit a declare que ie persiste par ce present codicille a mon testament olographe et de derniere volonté remis en depost entre les mains de $\mathrm{m} .{ }^{\theta}$ Louis chambalon no $^{\mathrm{re}}$ royal de la prevote de quebec, et autres ecrits, et que fesant le service a ma Comp. ${ }^{\circ}$ en la ville de montreal, ce n'est que pour eviter la continuation des violences, torts et domages que $\mathrm{mr}$ le marquis de vaudreuil gouverneur gen'al m'auroit fait souffrir pour se vanger contre moi a cause de la denonciation, et conviction que j'ay faite contre les sr arnaud comis de la comp. ${ }^{\circ}$ de la colonie au detroit pour vol et malversation des effets de la ditte Comp. ${ }^{\theta}$ lequel dit S..$^{\mathrm{r}}$ arnaud en gendre du S. ${ }^{\mathrm{r}}$ delotbinieres premier directeur, et icellui oncle de monsieur de vaudreuil par madame sa femme: et q'uen outtre $\mathrm{mr}$ le marquis de vaudreuil ne me detient en ce lieu contre les intentions du roy et ses ordres que pour favoriser les directeurs de la Comp. ${ }^{e}$ de ce pais, et pour me faire perdre par ressentiment la gratification que lad.

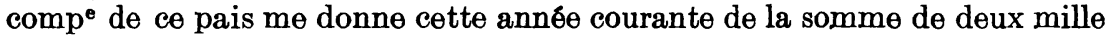
livres argent de france et ma nourriture et autres droicts mentionnez dans lacte passe entre les dits $\mathrm{S}^{\mathrm{rs}}$. directeurs de la ditte compe . et moy signé par feu mr le ch.r de calliere cy devant gouverneur gen. ${ }^{\text {al }}$ et par $\mathrm{mr}$ de beauharnois intendant: monsieur de vaudreuil n'a aucune raison prealable pour s'opposer a mon retour au fort pontchartrain ou ie commande puisque ie suis absous par le iugement de $\mathrm{mr}$ de beauharnois intendant touchant les faussetez qu'on m'a imposées aprez avoir subi une detention d'un an entier ayant eu la ville de quebec pour prison j'ay represente et fait mes remontrances a monsieur de vaudreuil sur ce que dessus par deux conferences que j'ay eu l'honneur d'avoir avec lui dans lesquelles aprez lui avoir expliqué et donné plus de quarante raisons deduites de mon placet du 17.e Juillet de la presente année il m'a dit que son plaisir êtoit tel que ie fisse le service ici a ma compagnie cêt pour quoy ie declare que si ie ne retourne pas au fort Séguin.

* Ce document nous a été fourni par notre collaborateur, M. Robert-Lionel 
pontchartrain ou j'ai l'honneur de comande par ordre du roy pour y continuer mes offices à la Comp..$^{\circ}$ de la colonie de ce païs ce n'est que par une force majeure et pour faire connoitre au roy mon entiere soumission et obeissance envers ceux qui representent en ce païs sa personne Sacrée puisque j'aurois êté bien fondé De me retirer du service par le tort, et l'iniustice qui m'est faite me voyant revoque d'un poste ou ie commande par ordre du roy $\mathrm{mr}$ le gouverneur gen.al $\mathrm{n}^{\prime}$ en uzant ainsi que par passion, et vangeance a cause du procez que j'ay eu avec les directeurs de la comp.e dans lequel ie l'ay recuse a Cause de sa parenté avec le s.r de L'otbiniere son dit oncle L'une de mes parties declarant et protestant que les service que ie ferai en ce lieu et ailleurs hors du fort pontchartrain ou ie suis detaché par ordre du roy ne pourra nuire ni préiudicier en rien aux pretentions que j'ay contre les directeurs de la comp. ${ }^{\ominus}$ de ce pais tant a cause de l'acte passé entre eux et moi que pour celles que iay contre eux, et les s. $^{\text {rs }}$ arnaud, et nolan comis malversateurs de la d. ${ }^{t}$ comp.$^{\circ} \mathrm{de}$ ce pais: esperant en oultre que sa Maj. to sera satisfaite de mon obeissance et qu'elle y pourvoira declarant que $i$ ay fait ce present codicille a mon d. $^{t}$ testament olographe pour me servir en tems et lieu, et devant qui il appartiendra. fait a montreal Le jour et an que dessus et venir le même jour en depost entre les mains du s. ${ }^{\mathrm{r}}$ adhemar no. ${ }^{\mathrm{re}}$ de cette ville du montreal dont jay retiré acte.

Lamothe cadillac

(Copie conforme à l'original conservé aux Archives judiciaires de Montreal). 